\title{
Assessment of fissionable material behaviour in fission chambers
}

\author{
O. Cabellos ，P. Fernández ，D. Rapisarda , N. García-Herranz \\ Instituto de Fusión Nuclear, Universidad Politécnica de Madrid, 28006 Madrid, Spain \\ Department of Nuclear Engineering, Universidad Politécnica de Madrid, 28006 Madrid, Spain \\ Laboratorio Nacional de Fusion, EURATOM-CIEMAT, 28040 Madrid, Spain
}

\section{A R T I C L E I N F O}

Keywords:

Fission chamber

BR2

DEMO

IFMIF

Uncertainty cross-sections

\begin{abstract}
A B S T R A C T
A comprehensive study is performed in order to assess the pertinence of fission chambers coated with different fissile materials for high neutron flux detection. Three neutron scenarios are proposed to study the fast component of a high neutron flux: (i) high neutron flux with a significant thermal contribution such as BR2, (ii) DEMO magnetic fusion reactor, and (iii) IFMIF high flux test module.

In this study, the inventory code $A C A B$ is used to analyze the following questions: (i) impact of different deposits in fission chambers; (ii) effect of the irradiation time/burn-up on the concentration; (iii) impact of activation cross-section uncertainties on the composition of the deposit for all the range of burn-up/irradiation neutron fluences of interest. The complete set of nuclear data (decay, fission yield, activation cross-sections, and uncertainties) provided in the EAF2007 data library are used for this evaluation.
\end{abstract}

\section{Introduction}

Fission chambers have been largely used in fission reactors to monitor and control the neutron flux. Nowadays, the feasibility of on-line neutron monitoring with fission chambers has been extended to other scientific interests such as neutron diagnostics in power fusion (ITER, DEMO) and in the IFMIF-Test Cell [1]. In nuclear waste or transmutation studies, the development of new fission chambers has been used to determine cross-sections measurements for thermal neutron-induced reactions [2].

The selection of the inner coating material of a fission chamber (FC) plays the most crucial role in order to assess the pertinence of sub-miniature fission chambers. Calculations concerning the isotopic composition and fission rates as a function of the neutron energy and fluence are necessary to assure the suitability of the chosen material.

Three neutron scenarios, see Fig. 1, of different degrees of hardness are proposed to study the behaviour of fissile materials: (i) the fast component of a high neutron flux with a significant thermal contribution common in a material testing reactor such as BR2 [3], (ii) the fusion power in a DEMO magnetic fusion reactor [4], and (iii) the fast neutron in the IFMIF high flux test module [4].

In order to select the fissile material for high energy neutron detection $(E>1 \mathrm{MeV})$, two main requirements must be fulfilled: (i) reduced burn-up for an integrated flux of $10^{22} \mathrm{n} / \mathrm{cm}^{2}$ and (ii) ability to measure neutron flux densities up to $10^{15} \mathrm{n} / \mathrm{cm}^{2} / \mathrm{s}$ in the energy range $1-50 \mathrm{MeV}$.

In a previous work [1], we selected a pure deposit of ${ }^{238} \mathrm{U}$ since it fulfills such requirements being commercially available, and therefore being good candidate for the HFTM/IFMIF, having a fission cross-section with an energy threshold in the MeV region. In this work, for a long-term performance up to a neutron fluence of $10^{22} \mathrm{n} / \mathrm{cm}^{2}$, we assess: (i) other realistic fissile deposits (see Table 1), (ii) the capability of spectral response for those selected materials, and (iii) the prediction of uncertainties due to the uncertainties in the neutron cross-section.

Table 1 shows the isotopic composition of some potential/ realistic deposits or solutions including some impurities. For calculations, we have taken a total amount of $10^{16}$ initial atoms. Consequently, the results presented here can be proportionally extrapolated to other deposits. The initial mass of the deposit corresponding to this initial amount of atoms is ranged from 3.85 to $4.0 \mathrm{~g}$.

The inventory ACAB code [5] has been used to take quantitatively into account the effect of daughter nuclides (produced in the coating during the irradiation)that may have high fission cross-section, therefore affecting the neutron flux measurements. The complete set of nuclear data (decay data, fission yield, activation cross-sections, and uncertainties) provided in the EAF2007 [6] library are used in this study. The 211 multi-group cross-section file covers the neutron energy range from $10^{-5} \mathrm{eV}$ to $60 \mathrm{MeV}$.

Section 2 is devoted to compute the reaction rates and saturation current delivered by a FC up to fluence of $10^{22} \mathrm{n} / \mathrm{cm}^{2}$. 


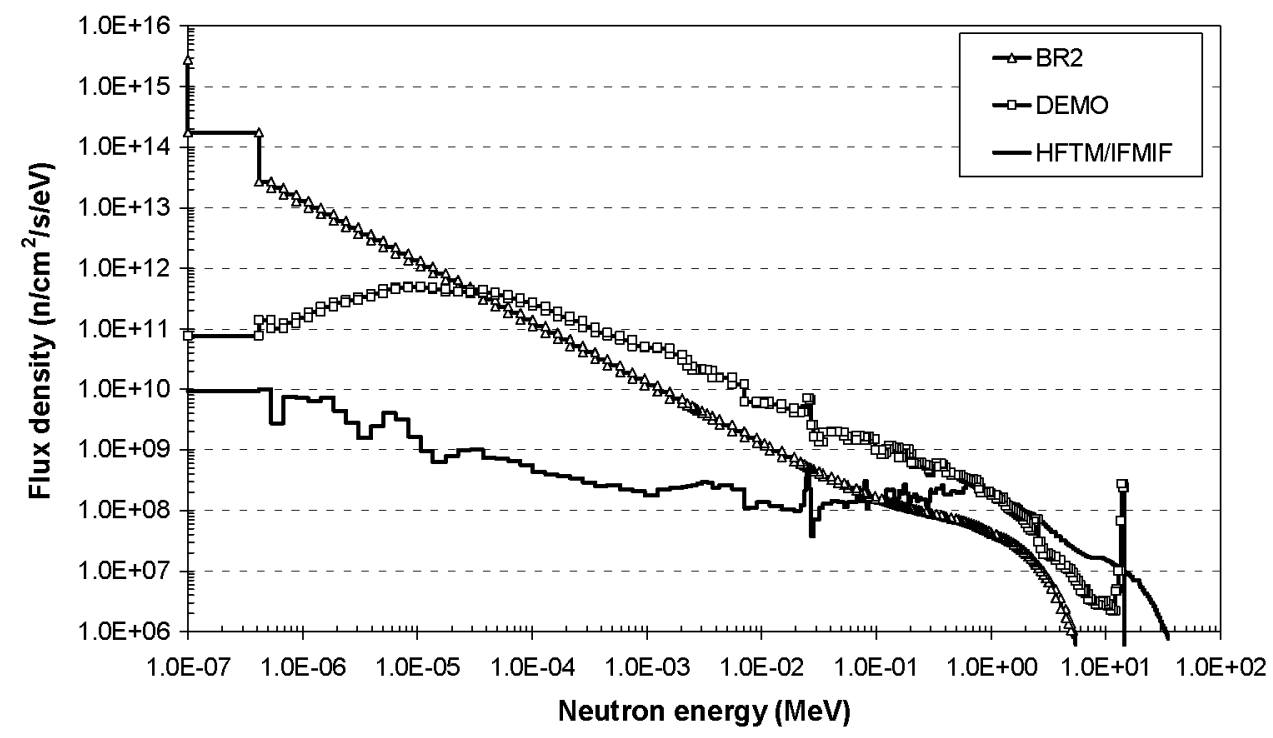

Fig. 1. Neutron spectra of different neutron environments in a VITAMIN-J+ (211) multi-group structure.

Table 1

Isotopic composition of the potential fissile deposit or solutions.

\begin{tabular}{|c|c|c|c|c|c|c|c|c|c|c|c|c|}
\hline \multirow[t]{2}{*}{ Deposit } & \multirow[t]{2}{*}{ Mass (g) } & \multirow{2}{*}{$\begin{array}{l}\text { Initial } \\
\text { activity (mCi) }\end{array}$} & \multicolumn{10}{|c|}{ Atomic percentage in $\%$} \\
\hline & & & ${ }^{232} \mathrm{Th}$ & ${ }^{235} \mathrm{U}$ & ${ }^{238} \mathrm{U}$ & ${ }^{237} \mathrm{~Np}$ & ${ }^{238} \mathrm{Pu}$ & ${ }^{239} \mathrm{Pu}$ & ${ }^{240} \mathrm{Pu}$ & ${ }^{241} \mathrm{Pu}$ & ${ }^{242} \mathrm{Pu}$ & ${ }^{244} \mathrm{Pu}$ \\
\hline Th232 & 3.85 & $4.23 \mathrm{E}-10$ & 100 & & & & & & & & & \\
\hline U235 & 3.90 & $8.22 \mathrm{E}-09$ & & 97 & 3 & & & & & & & \\
\hline $\mathrm{U} 238$ & 3.95 & $1.33 \mathrm{E}-09$ & & 0.04 & 99.96 & & & & & & & \\
\hline $\mathrm{Np} 237$ & 3.93 & $2.77 \mathrm{E}-06$ & & & & 100 & & & & & & \\
\hline Pu238 & 3.95 & $6.44 \mathrm{E}-02$ & & & & & 94.95 & 4.58 & 0.40 & 0.02 & 0.05 & \\
\hline Pu240 & 3.98 & $9.17 \mathrm{E}-04$ & & & & & 0.02 & 0.08 & 99.88 & & 0.02 & \\
\hline $\mathrm{Pu} 242$ & 4.02 & $1.59 \mathrm{E}-05$ & & & & & & & & & 100 & \\
\hline Pu242 \#1 & 4.02 & $9.08 \mathrm{E}-04$ & & & & & 0.214 & 0.116 & 0.172 & 0.180 & 99.274 & 0.044 \\
\hline $\mathrm{P} \cup 242$ \#2 & 4.02 & $1.64 \mathrm{E}-04$ & & & & & 0.004 & 0.005 & 0.022 & 0.035 & 99.932 & 0.002 \\
\hline
\end{tabular}

The sensitivity to the neutron spectrum is assessed as well. Then, in Section 3, the influence of different parameters on the FC behaviour is analyzed: radioactivity, xenon inventory, temperature, and deposit thickness. In Section 4, the impact of the uncertainties in activation cross-sections on the total fission rates and on the sensitivities to the neutron spectrum are performed, by using the Monte Carlo technique. The most contributing isotopes to the error in fission rates for deposits of Pu242\#2 and Np237 are shown. Finally, in Section 5, conclusions for the different realistic fissile deposits analyzed for a long-term performance are drawn.

\section{Computation of the reaction rates in a fission chamber and its sensitivity to neutron spectrum}

\subsection{Reaction rates and saturation current delivered by a fission chamber}

The isotopic inventory and fission rates are predicted by the $A C A B$ code. Originally, $A C A B$ was designed for activation and transmutation calculations in fusion and fission systems where neutron energy remains below $20 \mathrm{MeV}$. Recently, an updated version of $A C A B$ [5] is able to deal with high neutron energy $\left(E_{\mathrm{n}}>20 \mathrm{MeV}\right)$ using EAF2007 [6] cross-section libraries.

ACAB predicts the nuclide composition, $\mathbf{N}(t)=\left(N_{1}(t), N_{2}(t), \ldots\right.$, $\left.N_{\mathrm{N}}(t)\right)^{\mathrm{T}}$, of any material at time $t$, consisting of $N$ different nuclides. The set of differential equations which describe the evolution of $\boldsymbol{N}$ in a neutron field may be written in matrix notation as

$\frac{d N}{d t}=\mathbf{A} N$

where $\mathrm{A}$ is the $N$-by- $N$ transition matrix involving the averaged cross-section and decay values. Given $\mathbf{N}_{0}=\mathbf{N}(0)$ the initial nuclide density vector, the solution is $\mathbf{N}(t)=\exp$ $(\mathbf{A t}) \mathbf{N}_{0}$, assuming a constant spectrum and flux over the entire time step $[0, t]$.

The neutron spectrum can be separated in the following four energy-regions: thermal $(E<0.625 \mathrm{eV})$, epithermal $(0.625 \mathrm{eV}$ $<E<1.0 \mathrm{MeV}$ ), fast $(1 \mathrm{MeV}<E<20 \mathrm{MeV})$, and extended energy (above $20 \mathrm{MeV}$ ). Therefore, the total neutron flux can be decomposed into four components as follows from Eq. (2). Results for the neutron scenarios studied in this work are summarized in Table 2 .

$$
\begin{aligned}
\phi_{\text {total }} & =\sum_{g} \phi_{g}=\sum_{I=\text { ther,epi, } \ldots}\left(\sum_{g \in G_{I}} \phi_{g}\right) \\
& =\sum_{I} \phi_{I}=\phi_{\text {thermal }}+\phi_{\text {epi }}+\phi_{\text {fast }}+\phi_{\text {extended }}
\end{aligned}
$$

The collapsed one group cross-section with a multi-group neutron spectrum (see Fig. 1) is written as

$\frac{\sum_{g} \sigma_{g} \phi_{g}}{\sum_{g} \phi_{g}}=\frac{\sum_{g} \sigma_{g} \phi_{g}}{\phi_{\text {total }}}=\bar{\sigma}$ 
Total neutron flux and fraction of neutrons in four energy ranges for the three neutron scenarios.

\begin{tabular}{|c|c|c|c|c|c|}
\hline & \multicolumn{4}{|c|}{$\%$ neutrons having energies } & \multirow{2}{*}{$\begin{array}{l}\text { Total neutron } \\
\text { flux }\left(\times 10^{14}\right)\left(\mathrm{n} / \mathrm{cm}^{2} / \mathrm{s}\right)\end{array}$} \\
\hline & $E<0.625 \mathrm{eV}=E_{0}$ & $E_{0}<E<1 \mathrm{MeV}$ & $1 \mathrm{MeV}<E<20 \mathrm{MeV}$ & $E>20 \mathrm{MeV}$ & \\
\hline BR2 & 55.53 & 35.99 & 8.48 & - & 6.16 \\
\hline DEMO & 0.00 & 66.72 & 33.28 & - & 13.0 \\
\hline IFMIF/HFTM & 0.00 & 27.28 & 66.53 & 6.19 & 7.30 \\
\hline
\end{tabular}

Table 3

Collapsed one-group fission cross-section (barns), fraction of fissions for neutron energies above $1 \mathrm{MeV}$ (in \%) and fission/capture ratio.

\begin{tabular}{|c|c|c|c|c|c|c|c|c|c|}
\hline & \multicolumn{3}{|l|}{$\bar{\sigma}_{f i s s}(\mathrm{~b})$} & \multicolumn{3}{|c|}{ Fission fraction (in \%) $>1 \mathrm{MeV}$} & \multicolumn{3}{|c|}{ Fission/capture ratio } \\
\hline & BR2 & DEMO & HFTM & $\mathrm{BR} 2$ & DEMO & HFTM & BR2 & DEMO & HFTM \\
\hline Th232 & 0.01 & 0.1 & 0.2 & 99.6 & 100.0 & 100.0 & 0.001 & 0.1 & 2.0 \\
\hline U235 & 281.6 & 3.9 & 1.4 & 0.04 & 14.1 & 75.0 & 5.5 & 2.7 & 16.3 \\
\hline U238 & 0.0 & 0.3 & 0.5 & 99.1 & 99.8 & 99.8 & 0.004 & 0.1 & 7.5 \\
\hline $\mathrm{Np} 237$ & 0.2 & 0.7 & 1.5 & 66.7 & 82.6 & 88.3 & 0.002 & 0.2 & 8.4 \\
\hline Pu238 & 8.8 & 1.8 & 2.1 & 2.1 & 46.2 & 81.3 & 0.035 & 1.0 & 19.3 \\
\hline Pu239 & 482.8 & 4.0 & 1.9 & 0.03 & 18.1 & 76.0 & 2.2 & 1.9 & 25.4 \\
\hline Pu240 & 0.3 & 0.8 & 1.6 & 47.1 & 79.5 & 89.2 & 0.001 & 0.2 & 8.9 \\
\hline Pu241 & 564.5 & 5.7 & 1.8 & 0.02 & 11.2 & 72.9 & 2.8 & 4.2 & 8.5 \\
\hline Pu242 & 0.2 & 0.7 & 1.3 & 64.7 & 85.3 & 90.2 & 0.006 & 0.4 & 14.4 \\
\hline Pu243 & 98.2 & 2.91 & 1.3 & 0.11 & 18.5 & 76.6 & 1.98 & 9.8 & 28.2 \\
\hline Pu244 & 0.2 & 0.6 & 1.2 & 77.1 & 86.9 & 81.7 & 0.090 & 0.7 & 14.1 \\
\hline
\end{tabular}

where $\sigma_{g}$ is the averaged cross-section in the energy group- $g$, $\phi_{g}$ is the averaged neutron flux in group-g, and the sum is over all energy groups. It can be seen that $\bar{\sigma}$ depends only on the shape of the spectrum, and not on the total neutron flux. In Table 3 collapsed cross-sections for BR2, DEMO and IFMIF are shown.

The weighted cross-section in each group can be defined as

$\frac{\sum_{g \in G_{I}} \sigma_{g} \phi_{g}}{\sum_{g \in G_{I}} \phi_{g}}=\frac{\sum_{g \in G_{I}} \sigma_{g} \phi_{g}}{\phi_{I}}=\hat{\sigma}_{I}$

where $I \in\{$ thermal, epithermal, fast, extended $\}$ and $G_{l}$ is the set of groups in the various energy ranges.

An effective cross-section in each one of the energy regions [5,7] can also be defined as follows:

$\frac{\sum_{g \in G_{I}} \sigma_{g} \phi_{g}}{\sum_{g} \phi_{g}}=\frac{\sum_{g \in G_{i}} \sigma_{g} \phi_{g}}{\phi^{\text {total }}}=\bar{\sigma}_{I}$

It leads to the following relation:

$\sum_{g \in G_{I}} \sigma_{g} \phi_{g}=\hat{\sigma}_{I} \phi_{I}=\bar{\sigma}_{I} \phi^{\text {total }}$

The total fission reaction rate for a given neutron spectrum and for a given fissile deposit target can be computed as

$R_{\text {tot }}=\sum_{i} N^{i}(t) \sigma_{\text {fiss }}^{i} \phi_{\text {total }}$

with $N^{i}(t)$ the number of isotope $i$ in the deposit at time $t, \bar{\sigma}_{\text {fiss }}^{i}$ the one-group fission cross-section of the isotope $i$, and $\phi_{\text {total }}$ the total neutron flux intensity.

The corresponding fission reaction rates in each energy-region can be defined by

$R_{I}=\sum_{i} N^{i}\left(\sum_{g \in G_{I}} \sigma_{g}^{i} \phi_{g}\right)=\sum_{i} N^{i} \hat{\sigma}_{I}^{i} \phi_{I}=\sum_{i} N^{i} \bar{\sigma}_{I}^{i} \phi_{\text {total }}$

And, the total fission reaction rate is $R_{t o t}=\sum_{I} R_{I}$, where $R_{t o t}$ depends on the depletion (burn-up) of the fissile deposit with the neutron fluence, showing large differences with the neutron spectrum.

In the case of an impure deposit, we define the total fission rate as $R_{\text {tot }}=\sum_{k} \alpha_{k} R^{k}$, where $\alpha_{k}$ is the atomic percentage of the pure deposit $k$, and $R^{k}$ is the total fission rate of the pure deposit $k$.

For long-term performance, the burn-up/transmutation of the fissile deposit is important since it can directly affect the sensitivity of the chamber. The consumption of the fissile deposit is expected to drop exponentially as a function of the neutron fluence. Meanwhile, new fissile material is formed by capture reactions from the fissile material present in the initial composition of the deposit [8]. Fig. 2 shows the fission rate as a function of fluence for different pure and solution deposits in a high flux thermal neutron reactor such as BR2.

In Fig. 2, it can be seen that in BR2 only deposits with Pu242 remain stable up to fluences as high as $10^{22} \mathrm{n} / \mathrm{cm}^{2}$. At very low fluences, whereas the burn-up of U235 is still negligible, the deviation of pure Pu242 deposits starts due to the formation of ${ }^{243} \mathrm{Pu}$; this deviation can be compensated introducing a few quantities of fissionable isotopes (Pu242\#1 and Pu242\#2). At high fluences, for Pu242 deposits the contribution of ${ }^{245} \mathrm{Cm}$ to the total fission rate is very significant, varying from $\sim 1 \%$ to $\sim 80 \%$ between $10^{21}$ to $10^{22} \mathrm{n} / \mathrm{cm}^{2}$.

At a fluence of $10^{22} \mathrm{n} / \mathrm{cm}^{2}$ we have calculated the fission rate deviation with regard to the initial fission rate. In the particular case of BR2, large deviations were found except for Pu242\#1 deposit (as can be followed from Fig. 2). For DEMO, significant deviations were found in Pu240 (+20.9\%), U235 ( $-5.47 \%), \mathrm{U} 238$ $(+32.6 \%), \mathrm{Np} 237(6.6 \%)$ and $\mathrm{Th} 232(+65.2 \%)$. The rest of materials presented a deviation below $1 \%$. For the HFTM/IFMIF, deviations below $3.0 \%$ were predicted for all the studied materials.

The total fission reaction rates are directly related with the current delivered by the FC. When the chamber is working within the saturation domain, $I_{\text {sat }}=\Gamma \cdot R_{\text {tot }}$, where $\Gamma$ is the sensitivity factor of the detector depending only on the geometrical and physical features (gas composition, pressure, ...). 


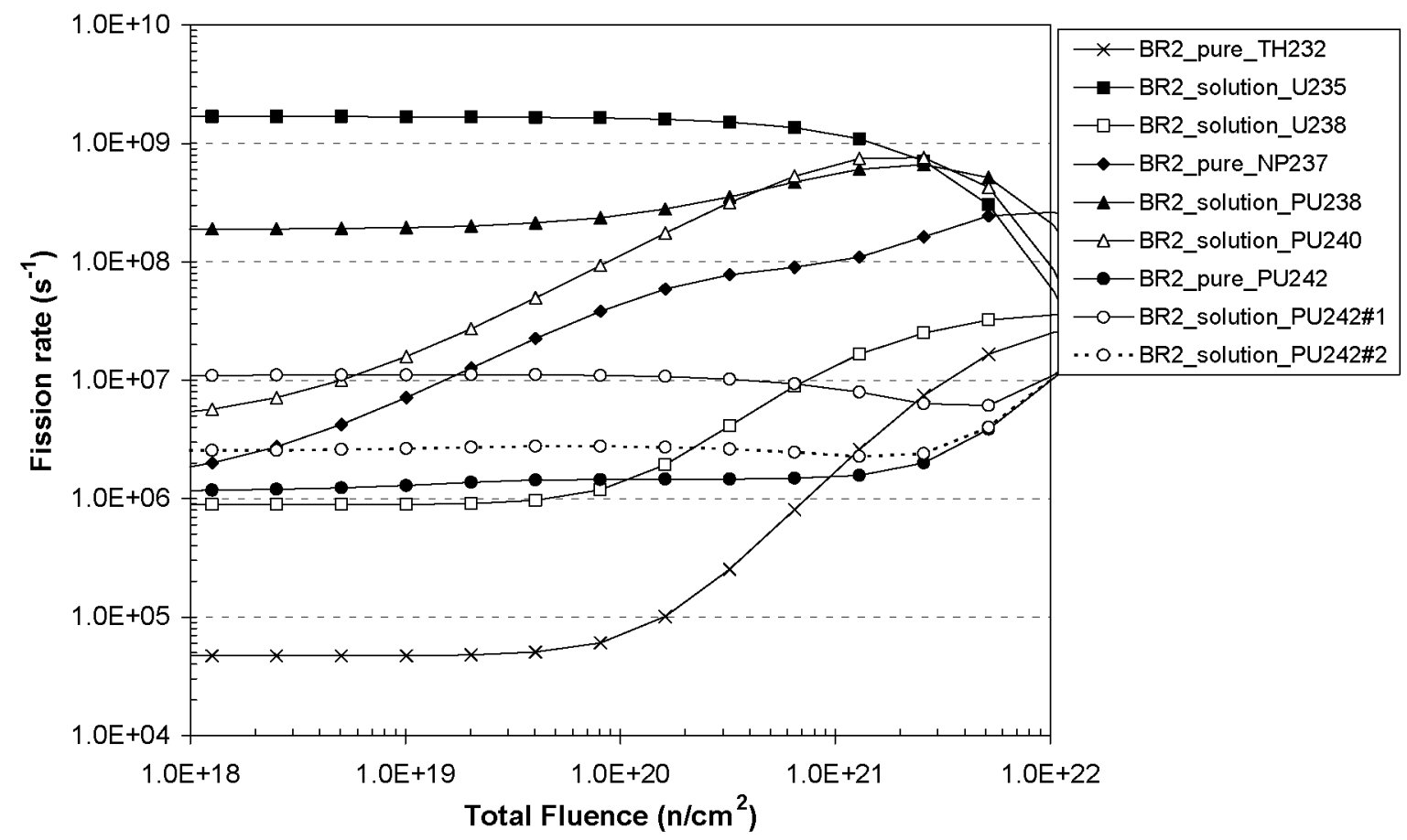

Fig. 2. Fission rates (fission/s) for the different pure and solution deposits in a typical high flux thermal neutron environment (BR2).

The behaviour of a FC is determined by its calibration curve (measured current versus polarization voltage). Depending on the voltage, three zones can be identified: recombination zone, saturation plateau, and avalanche zone. For a proper measurement of the radiation field the FC should work within the saturation zone, where all the primary created charges are collected.

The saturation plateau will gradually disappear and the transition between the recombination and the avalanche modes will be less visible [9] as the fission rates increase with the total fluence.

\subsection{Sensitivity of the saturation current with respect to the neutron spectrum}

In order to assess how the $I_{\text {sat }}$ of a FC (or the reaction rate of a given deposit) changes under flux variations, we define sensitivity coefficients according to Ref. [3]. These coefficients are good indicators of whether a given deposit would be efficient for neutron measurements.

If we define the relative change in the saturation current as

$\frac{\Delta I_{\text {sat }}}{I_{\text {sat }}}=\sum_{I=\text { ther,epi, } \ldots}\left(\left.\frac{\partial I_{s a t}}{\partial \phi_{I}}\right|_{\phi \neq \phi_{1}, \text { cte }} \frac{\phi_{I}}{I_{\text {sat }}}\right) \frac{\Delta \phi_{I}}{\phi_{I}}=\sum_{I=\text { ther }, \text { ep } i, \ldots} S_{I} \frac{\Delta \phi_{I}}{\phi_{I}}$.

Then, the sensitivity coefficient of the current with respect to a flux variation is

$S_{I}=\left.\frac{\partial I_{s a t}}{\partial \phi_{I}}\right|_{\phi \neq \phi_{I}, c t e} \frac{\phi_{I}}{I_{s a t}}=\left(\sum_{i} N^{i} \hat{\sigma}_{I}^{i}\right) \frac{\phi_{I}}{R_{t o t}}=\frac{R_{I}}{R_{t o t}}$

being $\sum_{i} S_{I}=1$.

These sensitivity coefficients depend on the isotopic composition, the neutron spectrum and the irradiation history or fluence. In addition, we can predict the sensitivity of an impure deposit formulated as $S_{I}=\sum_{k} \alpha_{k} S_{I}^{k} \beta_{k}$, where $S_{I}^{k}$ is the sensitivity of the pure deposit $k$, and $\beta_{k}$ is $R^{k} / R$, the relative fraction of fission rate of the pure deposit $k$.
In Fig. 3, the sensitivity coefficients to fast neutrons $\left(S_{\text {fast }}\right)$ for BR2 are shown. It can be seen that only the fissile deposit of Pu242\#1 and Pu242\#2 satisfies reasonable high values of $\mathrm{S}_{\text {fast }}$ $(\sim 30 \%)$ while remaining stable up to fluences as high as $3 \times 10^{21} \mathrm{n} / \mathrm{cm}^{2}$. These $S_{\text {fast }}$ values are similar to those obtained in Ref. [3]. The behaviour of these sensitivity coefficients as a function of the fluence has been investigated to deploy new methods taking into account the evolution of fissile deposit assessing on-line the fast-flux sensitivity [10].

\section{Other parameters having influence on the fission chamber behaviour}

\subsection{Total radioactivity}

The total radioactivity is needed both for waste management issues and to assess the impact on the background current. The progressive activation in both the structural pieces and the fissile deposit induces an increased secondary ionization processes in the FC so that some residual ionization can still be measured even without exposure to a neutron flux. It can be demonstrated that the activation structures has much more impact on the background current than the fission product activities comparing those parasitic currents in FCs with and without fissile deposit. The main effects of this residual ionization in FC are: (i) twisted calibration curve shapes and (ii) decreased voltage extension of the saturation regime [11]. Consequently, the total radioactivity prediction can help to better understand the FC behaviour for a long-term performance.

In this work, we have predicted the radioactivity only in the fissile deposit. It can be divided into two components: (i) the radioactivity released to the chamber by the fission fragments produced by the fission reaction (this term is proportional to the total fission reactions) (ii) and the one remaining in the coated material induced by transmutation reactions in the fissile deposit. 


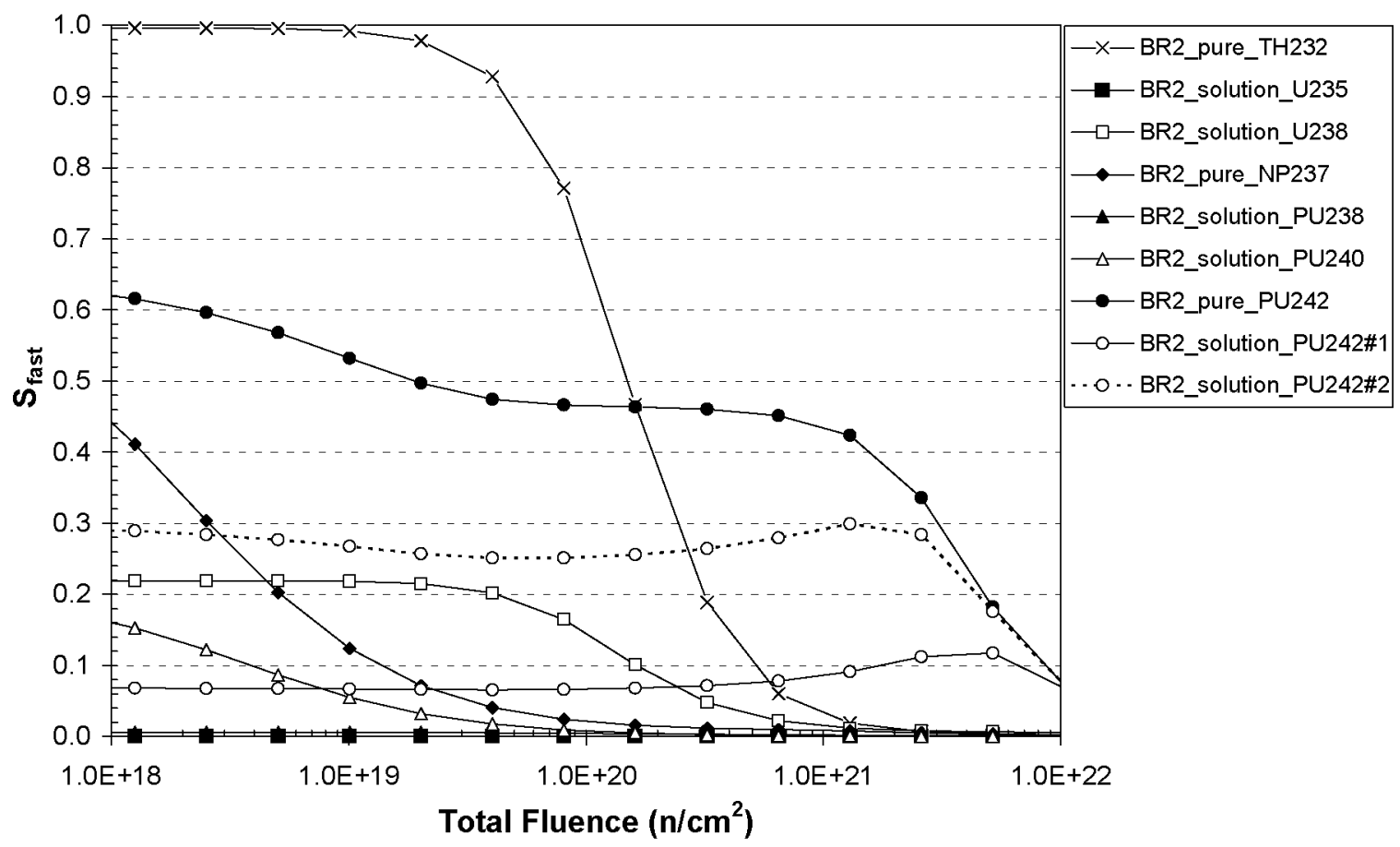

Fig. 3. Sensitivity to fast neutrons for initially different pure and solution deposits in a typical high flux thermal neutron environment (BR2).

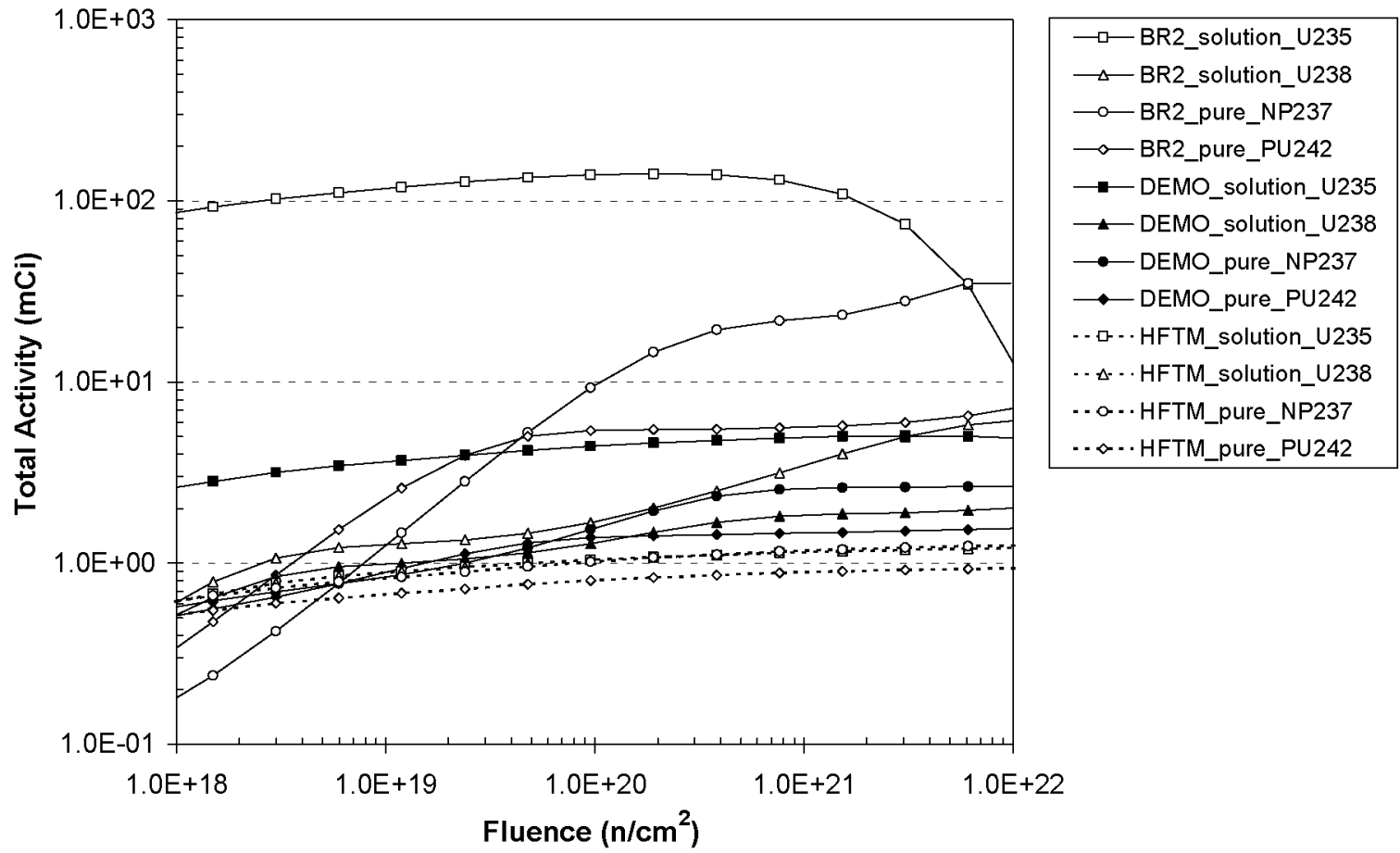

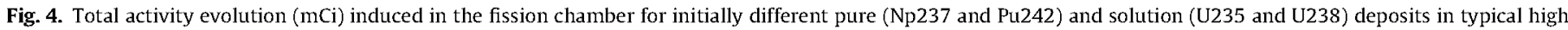
flux neutron environments.

Fig. 4 shows the evolution of activity induced in the FC for different fissile deposits in BR2, DEMO and HFTM/IFMIF environments. For the deposits U238, Pu242, Np237, and Th232 the main component of the radioactivity will remain in the deposit due to the formation of new radioactive material by $(n, \gamma)$ reactions $\left({ }^{243} \mathrm{Pu}\right.$ from ${ }^{242} \mathrm{Pu},{ }^{239} \mathrm{U}$ from ${ }^{238} \mathrm{U},{ }^{238} \mathrm{~Np}$ from ${ }^{237} \mathrm{~Np}$ and ${ }^{233} \mathrm{Th}$ and ${ }^{233} \mathrm{~Pa}$ from ${ }^{232} \mathrm{Th}$ ). For the deposits of U235, Pu238 and Pu240, most of the radioactivity is released to the $\mathrm{FC}$ by the fission products.

\subsection{Xenon inventory}

Fission chambers operating at high neutron fluences are usually filled with inert gases at high pressure (e.g. argon) [11]. However, the addition of contaminants with low ionization potential (e.g. xenon) involves a shift of avalanche towards lower voltages, having catastrophic consequences on the size of saturation plateau [9]. It has been experimentally observed that 
in standard FC with Argon at a pressure of 1 bar, the contamination of $0.1 \%$ in Xe shifts the voltage at the beginning of avalanche by $20 \mathrm{~V}$.

Xenon is one of the most abundant fission products and therefore its concentration can be predicted by $A C A B$ code during the irradiation time. Fig. 5 shows the total isotopic prediction of xenon due to fission reactions in the deposit of the FC. Levels higher than $10^{16}$ atoms of xenon are obtained in deposits of U235, Np237, Pu238, Pu240 in BR2 reactor. Xenon from $10^{15}$ to $10^{16}$ atoms were found for U238 and Th232 deposits in BR2, U235 and Pu238 for DEMO and Pu238 for HFTM (some of these deposits not shown in the figure).

As an example of the importance of Xe prediction in a typical fission chamber, CFUR43 detector by PHOTONIS was selected. Although the geometrical characteristics of the CFUR43 are not totally known, we have taken its geometrical and physical features from [9,12]: Ar as internal gas, pressure at 1 bar, interelectrode gap of $0.25 \mathrm{~mm}$ (the suggested value is between 0.2 and $0.3 \mathrm{~mm}$ [12]) and length of $17.55 \mathrm{~cm}\left(z_{\max }: 0.71 \mathrm{~cm} ; z_{\min }\right.$ : $-1.045 \mathrm{~cm}$ [9]). These values lead, considering a cylindrical volume, to an Ar mass of about $4.2 \times 10^{-8} \mathrm{~kg}$; i.e. $6.34 \times 10^{17} \mathrm{Ar}$ atoms.

As we can see in Fig. 5, the level of neutron fluence to be considered for Xe contamination above $0.1 \%$ depends on the fissile deposit; the lowest is for U235 in BR2 with a fluence of $\sim 6 \times 10^{19} \mathrm{n} / \mathrm{cm}^{2}$.

\subsection{Temperature effect}

In this section, we assess the temperature effect on the total fission rates for different deposits irradiated in BR2 [13]. In this reactor, the temperature dependence can be explained by the fact that the thermal neutron flux, described by the Maxwell distribution, becomes harder with increasing temperature, and consequently the one-group cross-sections will change.
In order to evaluate the temperature effect, two sets of calculations have been performed:

(i) In the first set, named "BRANCHING" cases, the reference temperature of $325 \mathrm{~K}$ used in Ref. [3] is instantaneously changed to $350 \mathrm{~K}$ at each irradiation time. Assuming a constant total neutron flux, the differences in the total fission rates are due to the different effective one-group fission crosssections (each one collapsed with a different thermalized neutron component described by a Maxwell distribution as a function of the temperature).

(ii) In the second set, named spectral history cases, "SH", we keep the temperature at $350 \mathrm{~K}$ during the whole irradiation time, so not only the one-group fission cross-sections change, but also the atomic concentration.

Such temperature effects would produce a relative fission rate difference $(\Delta R)$ showed in Fig. 6. For U235 deposit, a relative difference of $-2 \%$ is found when changing instantaneously the temperature from 325 to $350 \mathrm{~K}$ (branching case up to $10^{22} \mathrm{n} / \mathrm{cm}^{2}$ ). The same difference is found for the spectral history case up to $10^{20} \mathrm{n} / \mathrm{cm}^{2}$, but this deviation is reversed and it reaches a value of $+4 \%$ at $10^{22} \mathrm{n} / \mathrm{cm}^{2}$. At this high fluence, this difference in the spectral history case is due to a relative increase of the fission rate of $+5 \%$ in the isotope U235 and a decrease of $-2.2 \%$ in the isotope Pu239. In the case of Pu242 deposit, differences between branching and spectral history cases are nearly the same.

\subsection{Deposit thickness}

In this section, the importance of the thickness of the deposit in the FC behaviour has been studied, taking into account the selfshielding phenomenon and the fission product trapping within the deposit.

First, to analyze the self-shielding phenomenon (that is, less neutron absorption in the inner layers of the deposit), we predict

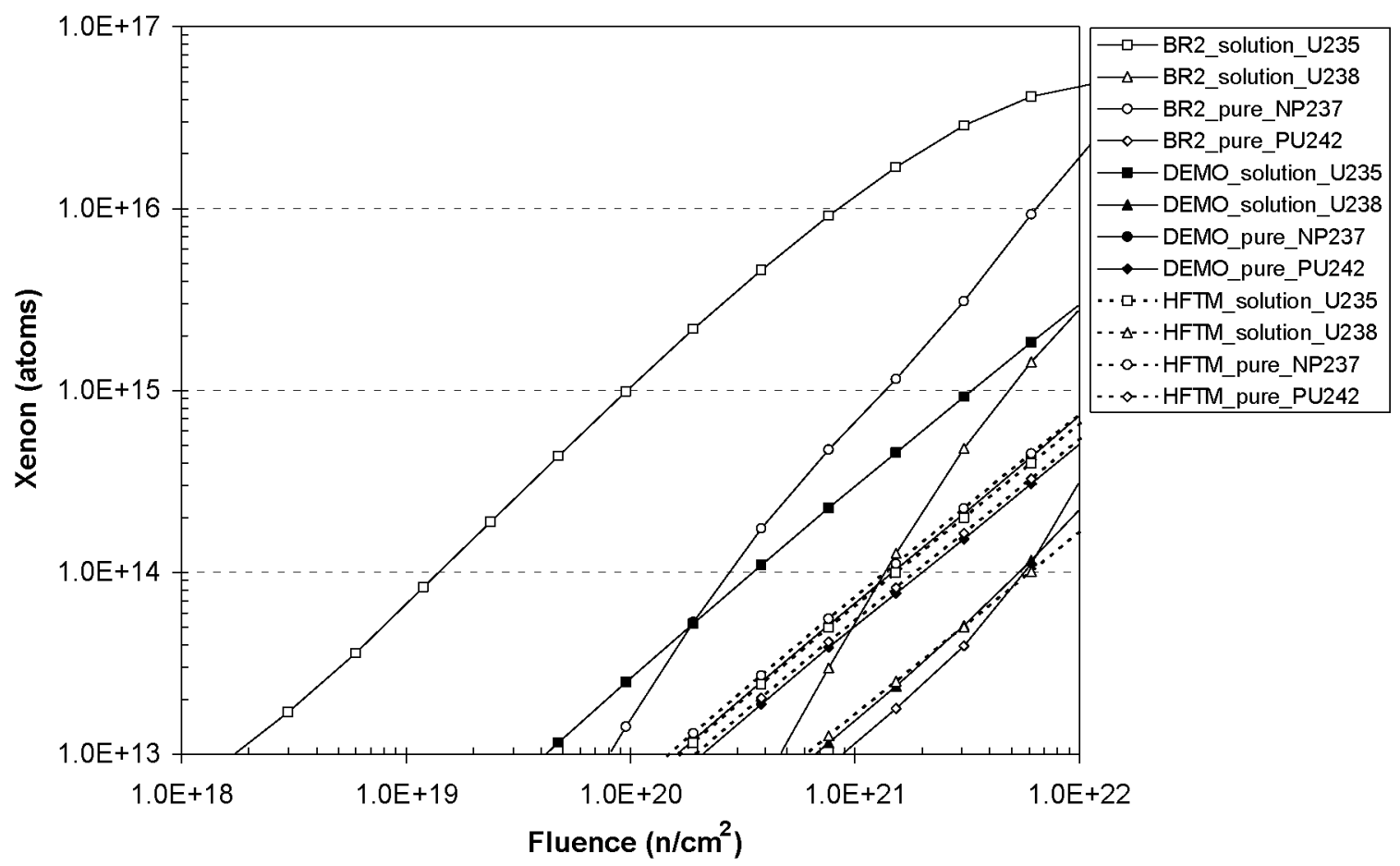

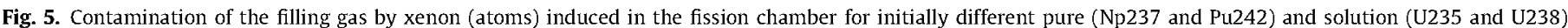
deposits in typical high neutron flux environments. 


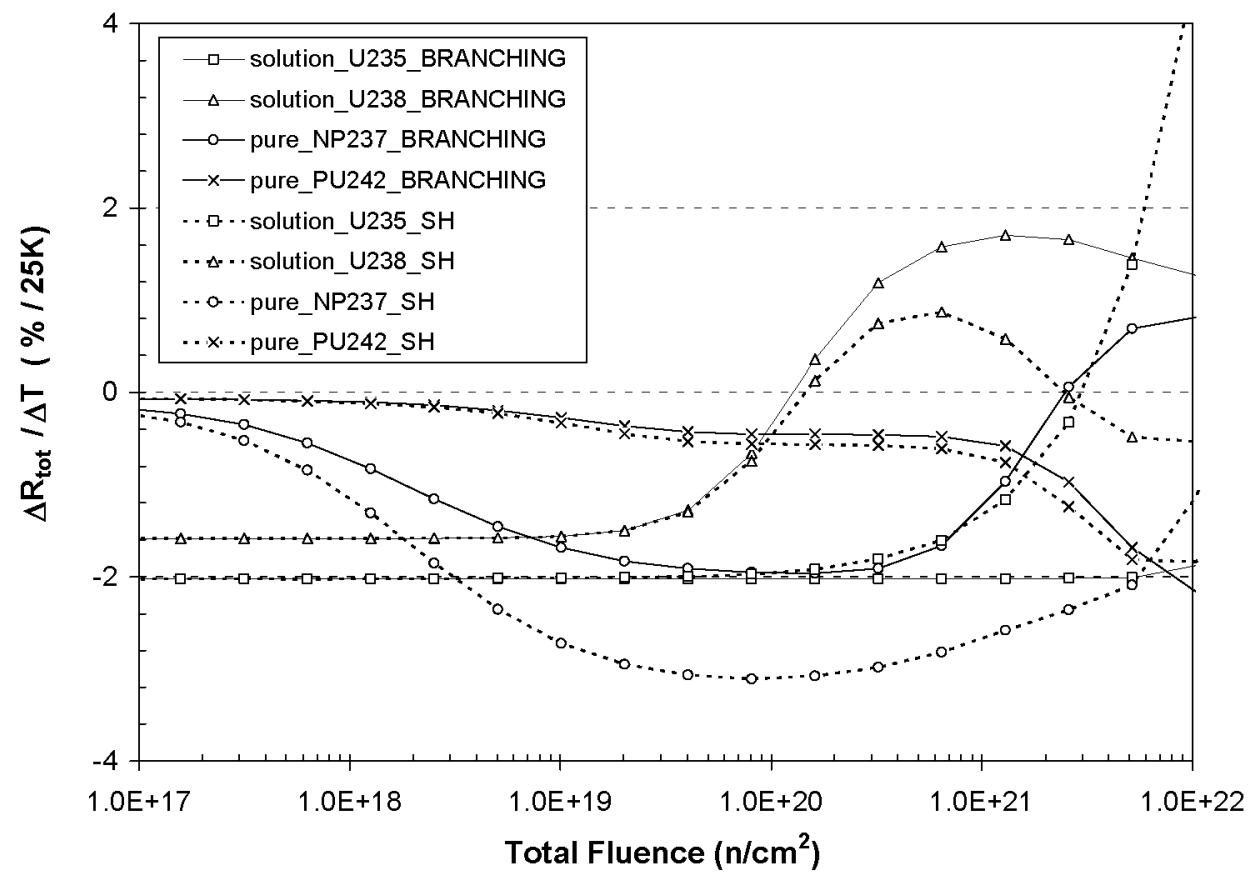

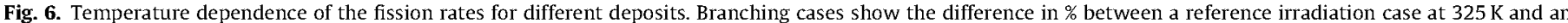
instantaneous change in the temperature of $+25 \mathrm{~K}$. In the spectral history cases, we compare the nominal case at $325 \mathrm{~K}$ with a modified irradiation case at $350 \mathrm{~K}$.

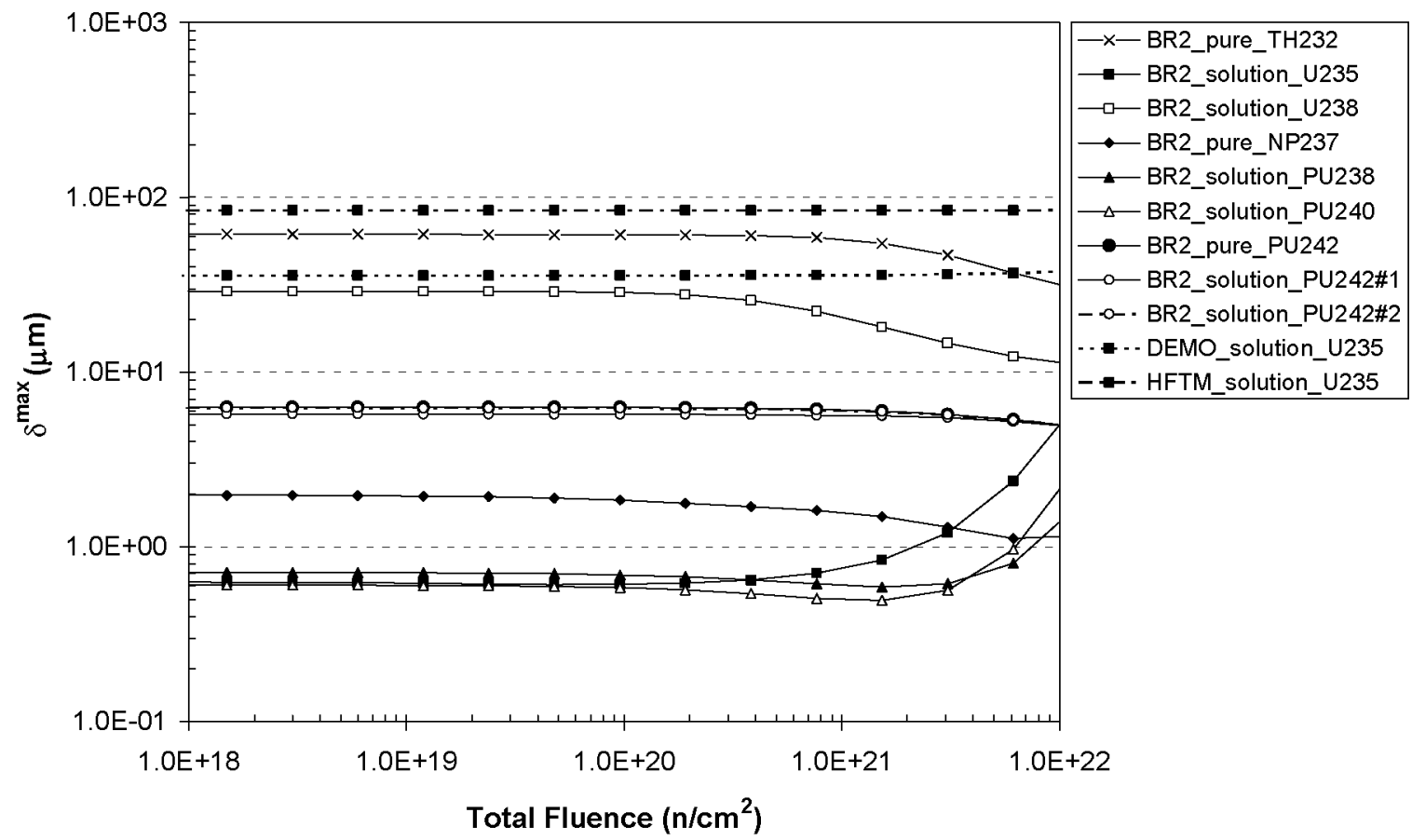

Fig. 7. Predicted maximum thickness assuming an interaction probability $(\varepsilon)$ of 0.001 .

the interaction probability in the deposit thickness $(\delta)$ as $\Sigma_{\text {total }} \cdot \delta$, being $\Sigma_{\text {total }}=\Sigma_{i=1}^{N} \sigma_{\text {total }}^{i} N^{i}$ the macroscopic cross-section in $\mathrm{cm}^{-1}$, $\sigma_{\text {total }}^{i}$ the total microscopic cross-section in $\mathrm{cm}^{2}, N^{i}$ is the atomic density (atm/ $/ \mathrm{cm}^{3}$ ) for isotope $i$, and $N$ is the total number of isotopes in the deposit.

For a density of the fissile deposit of $\rho^{\text {deposit }}\left(\mathrm{g} / \mathrm{cm}^{3}\right)$, we can write $\Sigma_{\text {total }}$ as

$\Sigma_{\text {total }}=\rho^{\text {deposit }} N_{A} \frac{\sum_{i=1}^{N} \alpha^{i} \cdot \sigma_{\text {total }}^{i}}{\sum_{i=1}^{N} \alpha^{i} \cdot A^{i}}$ where $\alpha^{i}$ is the fraction for isotope $i$ in the deposit, $N_{A}$ is the Avogadro number, and $A^{i}$ is the atomic weight of isotope $i$.

Assuming an interaction probability, $\varepsilon$, we predict the maximum thickness as $\delta^{\max }<\varepsilon / \Sigma_{\text {total }}$. It can be seen (in Fig. 7) that with a typical thickness of $50 \mathrm{~nm}$ this effect is negligible in all deposits and for neutron fluences up to $10^{22} \mathrm{n} / \mathrm{cm}^{2}$.

Second, we have assessed the fission product trapping within the deposit (auto-absorption). We can roughly estimate the total kinetic energy of any fragment assuming that a fragment's initial kinetic energy is inversely proportional to its mass, and using a empirically defined dependence of the average kinetic energy of 


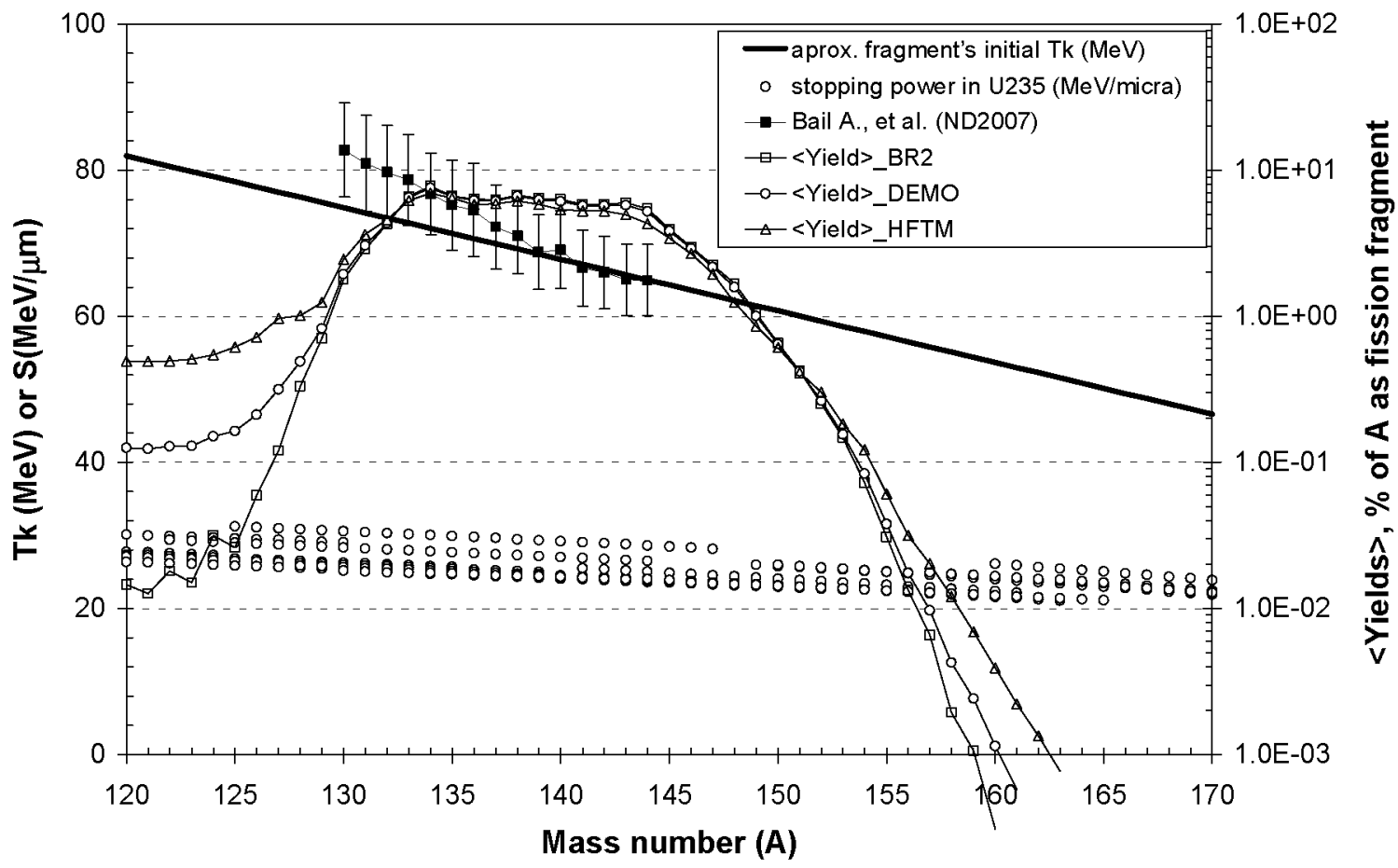

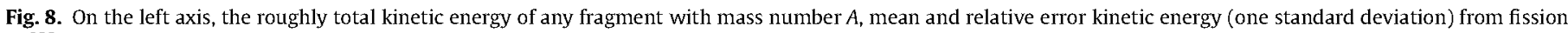

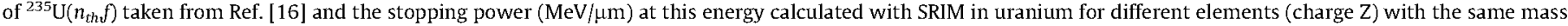
number. On the right axis, the effective fission product yield for ${ }^{235} \mathrm{U}$ collapsed by $\mathrm{ACAB}$ code for different neutron spectra.

fission fragments on the mass and the charge of a fissioning nucleus [14]: $\left\langle T_{k}(\mathrm{MeV})\right\rangle=0.1178 Z^{2} / A^{1 / 3}+5.8$. Thus, the trapping of heavy fission products can be neglected, if for such deposit thickness the stopping power is not significant. The stopping power $(\mathrm{MeV} / \mu \mathrm{m})$ at this energy is predicted by SRIM code [15]. In this case, we can considerer that all the fission products emitted toward the gas reach the gas chamber [9]. All of these values are shown in Fig. 8.

Moreover, Fig. 8 shows the effective fission product yield $\langle\gamma\rangle$, defined as the spectrum-averaged fission yield for formation of nuclei $i$ by fission in the nuclei $j$. This value is needed to predict the generation of fission products in the FC and it can be expressed as

$\left\langle\gamma_{j, i}\right\rangle=\frac{\int_{0}^{\infty} \gamma_{j, i}(E) \sigma_{f, j}(E) \phi(E) d E}{\int_{0}^{\infty} \sigma_{f, j}(E) \phi(E) d E}$

where $\gamma_{j, i}$ is the probability that a type-i nuclide will be formed as a fission product by absorption of a neutron of energy $E$ by a nuclide of type-j, and $\sigma_{f, j}$ is the microscopic fission cross-section of type- $j$ nuclei for neutrons of energy $E$.

\section{Impact of activation cross-section uncertainties}

It is well known that the activation cross-section uncertainties remain high for some isotopes, and this fact may cause significant uncertainties in the isotopic inventory prediction. In this section, we calculate uncertainty estimates of the actinide inventory, and response quantities such as fission rate or sensitivities due to the current uncertainties in the activation cross-section library, EAF2007/UN.

The uncertainties provided by EAF2007/UN are the most complete uncertainty library in regards to the number of nuclides considered for inventory uncertainty prediction. The information included in these uncertainty files refers to the relative error in the cross-section, denoted hereafter as $\Delta_{E A F}$ (in fact, the information included in these files is $\Delta_{\mathrm{FAF}}^{2}$, where $I$ is the energy group). Uncertainties $\boldsymbol{A}_{I, E A F}$ are provided in a four-energy group structure (up to $60 \mathrm{MeV}$ ) for non-threshold reactions and in one/two energy group for threshold reactions. Note that the energy group boundaries are different for each reaction and isotope. It is assumed that errors in cross-sections included in an energy group are $100 \%$ correlated, and that the errors between different energy groups are uncorrelated. It can be seen that these conservative uncertainty values, $\Delta_{I, E A F}$, as three times the experimental standard deviation of cross-section, that is $A_{I, E A F}=3^{*} A_{I, E X P}$ in order to represent a $99.73 \%$ confidence limit

In order to measure the importance of these uncertainties we define the corresponding total relative experimental error $\left(\Delta_{E X P}\right)$. This value is obtained collapsing the uncertainty data for a particular neutron spectrum according with this formula [5,7]

$A_{E X P}^{2}=\sum_{I} A_{I, E X P}^{2}\left(\frac{\bar{\sigma}_{I}}{\bar{\sigma}}\right)^{2}$

where $\bar{\sigma}_{I}$ is the effective cross-section in one of the energy groups and $\bar{\sigma}$ the one-group cross-section. For inventory calculations, this procedure to derive one-group uncertainty data guaranties that the uncertainty using different group structure remains constant [17]. In Table 4 , the total relative error for $(n, \gamma)$ and ( $n$, fission) reactions of some important isotopes are computed. It can be seen that for BR2 and DEMO, fission reaction has larger uncertainty than capture reaction, and for HFTM/IFMIF similar errors were found. ${ }^{243} \mathrm{Pu}$ and ${ }^{238} \mathrm{~Np}$ have the largest uncertainty values both for fission and capture reactions.

To analyse how cross-section uncertainty is transmitted to the vector $\mathbf{N}$ and to other response quantities obtained as a function of $\mathbf{N}$ (e.g., $\left.R_{t o t}, S_{f a s t}, \ldots\right)$ we define a random vector $\sigma=\left(\sigma_{1}, \sigma_{2}, \ldots\right.$, $\sigma_{M}$ ), where $M$ is the total number of activation cross-sections involved in the problem. Let us assume that the other parameters 
(decay values, fission yields,...) are known and constant (not random), and that the analysis is carried out for a fixed time $t$. Each isotope $N_{i}$ is a function of the random vector $\sigma, N_{i}=N_{i}(\sigma)$ where $t$ will not be included for simplicity.

The inventory code $A C A B$ uses two different approaches to assess the impact of nuclear data uncertainties on the radionuclide inventory: sensitivity/uncertainty (S/U) analyses and Monte Carlo uncertainty (MC/U) technique.

\subsection{Sensitivity/uncertainty analysis}

Let $\bar{\sigma}$ be the estimated cross-section vector and $\left.\mathbf{N}(\bar{\sigma})=\left(\mu_{1}, \mu_{2}, \ldots, \mu_{N}\right)\right)$ the solution of Eq. (1) at this point. First order Taylor series provides a means of approximating $N_{i}(\sigma)$ about $\bar{\sigma}$

$N_{i}(\sigma) \approx N_{i}(\bar{\sigma})+\sum_{j=1}^{M}\left[\frac{\partial N_{i}}{\partial \sigma_{j}}\right]_{\bar{\sigma}}\left(\sigma_{j}-\bar{\sigma}_{j}\right)$

Table 4

Total relative experimental error (in \%) for capture and fission cross sections from EAF2007/UN.

\begin{tabular}{|c|c|c|c|c|c|c|}
\hline & \multicolumn{3}{|c|}{ Fission } & \multicolumn{3}{|c|}{ Capture } \\
\hline & BR2 & DEMO & HFTM & BR2 & DEMO & HFTM \\
\hline Th232 & 16.6 & 16.7 & 13.7 & 2.5 & 3.8 & 15.4 \\
\hline U235 & 3.1 & 7.0 & 15.1 & 3.0 & 4.8 & 15.5 \\
\hline U238 & 16.6 & 16.7 & 13.9 & 2.8 & 3.3 & 13.5 \\
\hline $\mathrm{Np237}$ & 15.8 & 16.6 & 15.4 & 3.2 & 7.6 & 15.0 \\
\hline $\mathrm{Np238}$ & 46.7 & 24.7 & 30.7 & 33.0 & 24.5 & 32.3 \\
\hline Pu238 & 5.4 & 12.5 & 15.6 & 3.9 & 10.0 & 15.9 \\
\hline Pu239 & 3.3 & 6.0 & 15.3 & 4.5 & 8.6 & 14.3 \\
\hline Pu240 & 14.0 & 16.2 & 14.9 & 2.5 & 3.5 & 7.8 \\
\hline Pu241 & 3.3 & 8.8 & 15.1 & 3.0 & 8.0 & 16.3 \\
\hline $\mathrm{Pu} 242$ & 14.8 & 16.5 & 15.2 & 8.0 & 8.0 & 14.0 \\
\hline Pu243 & 118.4 & 47.67 & 59.5 & 274.6 & 23.6 & 32.4 \\
\hline Pu244 & 16.5 & 16.6 & 15.2 & 15.1 & 23.9 & 32.6 \\
\hline
\end{tabular}

which can be written as

$\frac{N_{i}(\sigma)-\mu_{i}}{\mu_{i}} \approx \sum_{j=1}^{M} \rho_{i j} \frac{\left(\sigma_{j}-\bar{\sigma}_{j}\right)}{\bar{\sigma}_{j}}$

where

$\rho_{i j}=\frac{\bar{\sigma}_{j}}{\mu_{i}}\left[\frac{\partial X_{i}}{\partial \sigma_{j}}\right]_{\bar{\sigma}}$

is known as the sensitivity coefficient. The value $e_{i}=N_{i}(\sigma)-\mu_{i} / \mu_{i}$ denotes the relative change (error) in the amount of nuclide $i$ due to relative changes (errors) in cross-sections equal to $\varepsilon_{j}=\left(\sigma_{j}-\bar{\sigma}_{j}\right) / \bar{\sigma}_{j}$. For the random vector $\varepsilon=\left[\varepsilon_{1}, \varepsilon_{2}, \ldots, \varepsilon_{M}\right]^{\mathrm{T}}$, the $M$-by- $M$ relative covariance matrix or fractional error matrix is $M=E\left[\varepsilon \varepsilon^{\mathrm{T}}\right] . M$ is a diagonal matrix representing relative uncertainties in the activation cross-sections from EAF2007/UN.

Eq. (13) gives a natural method for obtaining an approximation for the fractional error of $e=\left(e_{1}, e_{2}, \ldots, e_{N}\right)^{\mathrm{T}}$. Let $S$ be the $N$-by- $M$ matrix containing the sensitivity coefficients, i.e.,

$S=\left[\begin{array}{cccc}\rho_{11} & \rho_{12} & \cdots & \rho_{1 M} \\ \rho_{21} & \rho_{22} & \cdots & \rho_{2 M} \\ \vdots & \vdots & \ddots & \vdots \\ \rho_{N 1} & \rho_{N 2} & \cdots & \rho_{N M}\end{array}\right]$

then $e \approx S \varepsilon$ and taking expectations on both sides $E[e] \approx S E[\varepsilon]$ and the $N$-by- $N$ variance matrix of $e$ is $M_{e} \approx S M S^{T}$.

To deal with response functions directly dependent on the isotopic inventory the sensitivity/uncertainty formalism is straightforward. In the most simple case predicting fission rate, $R$ as: $R=N \sigma_{f} \phi_{\text {total }}$, the first Taylor approximation predicts the relative error of $R\left(\varepsilon_{R}\right)$ by: $\varepsilon_{R}^{2}=\varepsilon_{N}^{2}+\varepsilon_{\sigma_{\text {fass }}}^{2}+2 \varepsilon_{N} \varepsilon_{\sigma_{\text {fis }}}$. According with this formula, it can be concluded that the main source of uncertainty in fission rate is due to the uncertainty in fission cross-sections. Uncertainty in the concentration could play an important role at high neutron fluences.

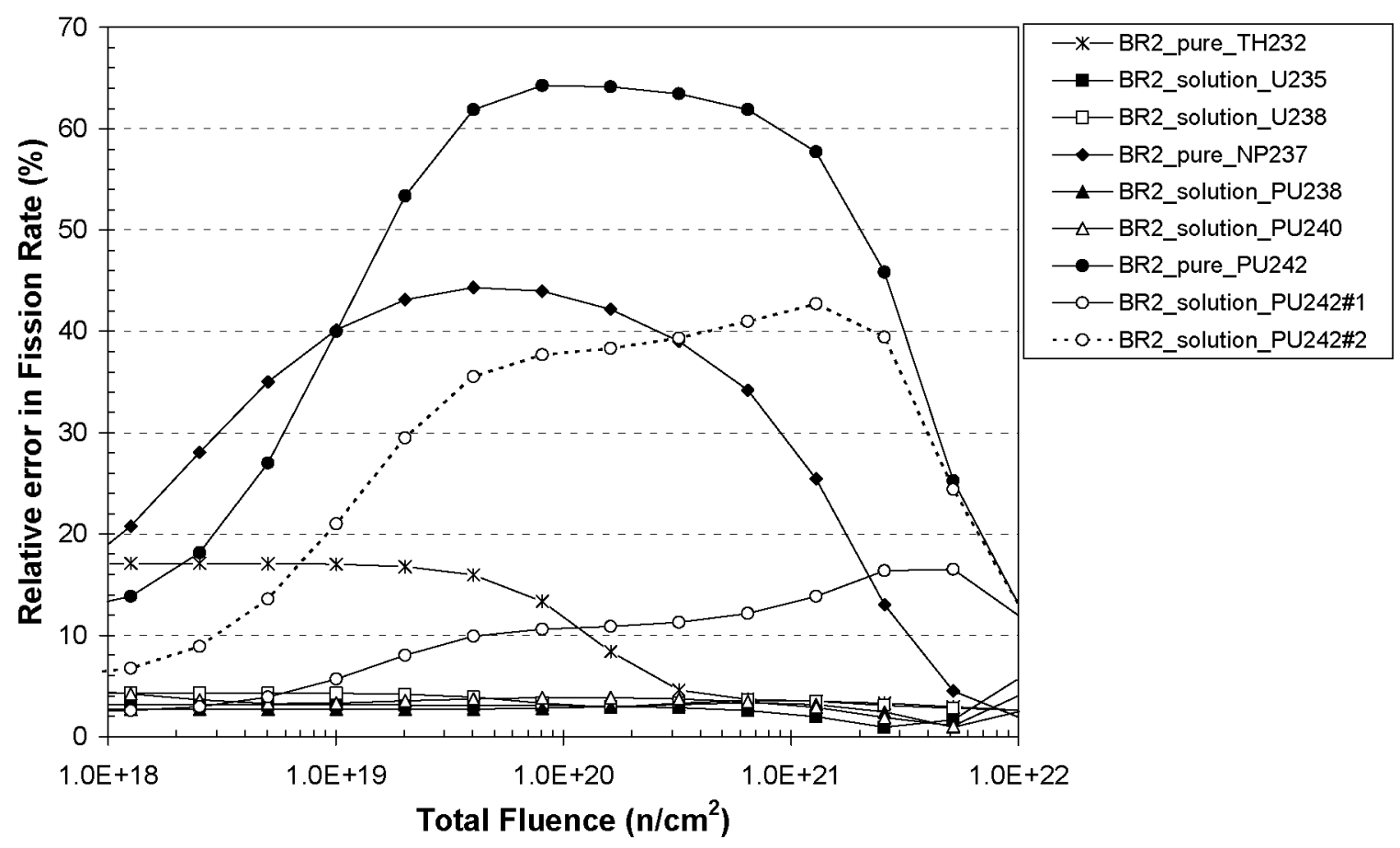

Fig. 9. Relative error in fission rates (in \%) for different pure and solution deposits in a typical high flux thermal neutron environment (BR2). 
In a general case

$\varepsilon_{R}^{2}=\sum_{j=1}^{F} \alpha_{j}^{2}\left(\sum_{i=1}^{M} \varepsilon_{N_{i}, \sigma_{i}}^{2}\right)+\sum_{j=1}^{F} \alpha_{j}^{2} \varepsilon_{\sigma_{f i s, i}}^{2}+2 \sum_{j=1}^{F} \alpha_{j} \varepsilon_{\sigma_{f i s s, j}}\left(\sum_{i=1}^{F} \alpha_{i} \varepsilon_{N_{i}, \sigma_{f i s, i}}^{2}\right)$

where $\alpha_{j}$ is the fraction of isotope $j$ contributing to the total fission rate. And $F$ is the total number of fissile isotopes.

\subsection{Monte Carlo method}

Here, the implemented Monte Carlo (MC) method in $A C A B$ code is applied to FC uncertainty analysis, showing its capability to deal with overall cross-section uncertainty data. The MC method is based on simultaneous random sampling of all the cross-sections involved in the problem, where uncertainties are taken from EAF2007/UN. Authors of the EAF library have noted

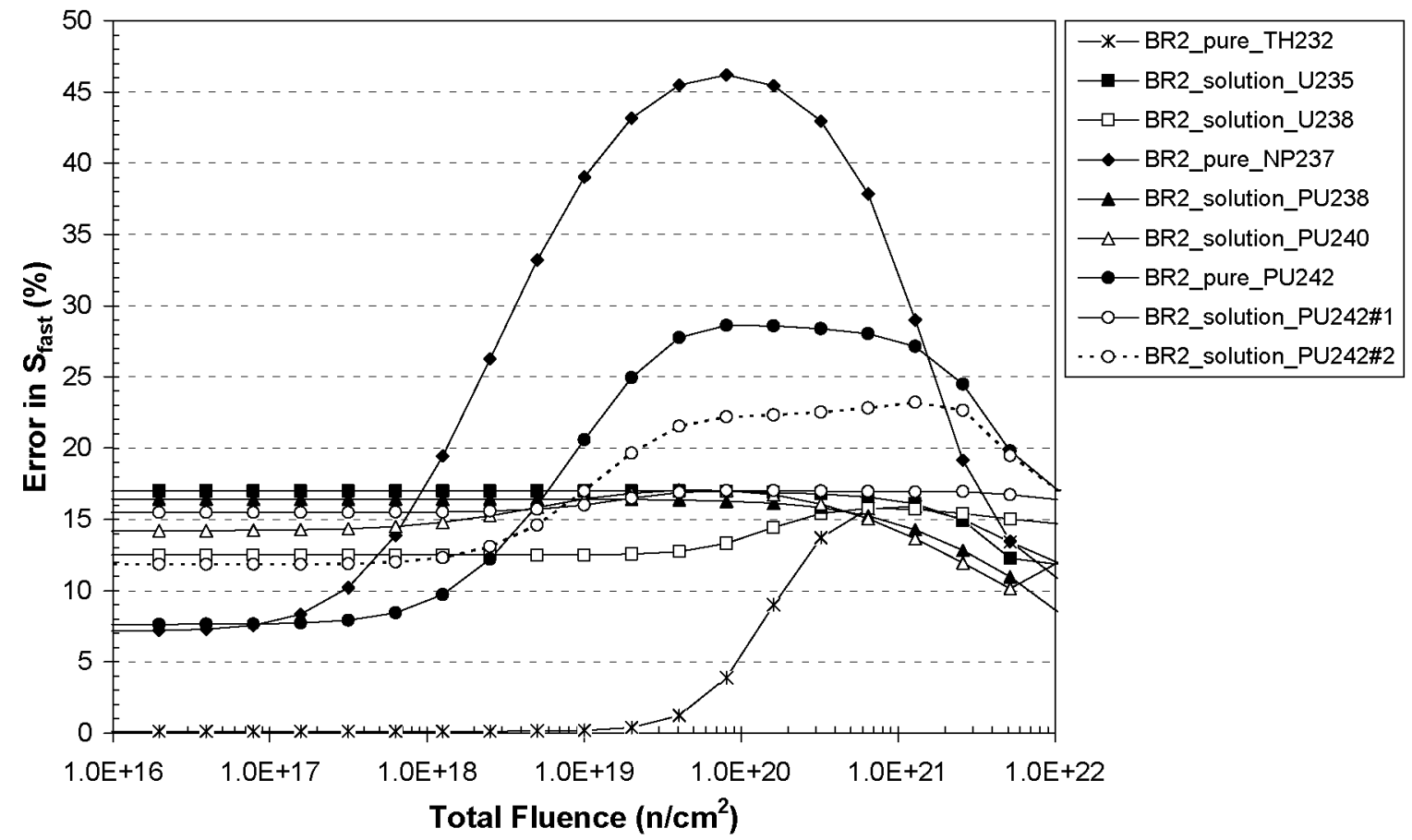

Fig. 10. Relative error in Sensitivity to fast neutrons (in \%) for different pure and solution deposits in a typical high flux thermal neutron environment (BR2).

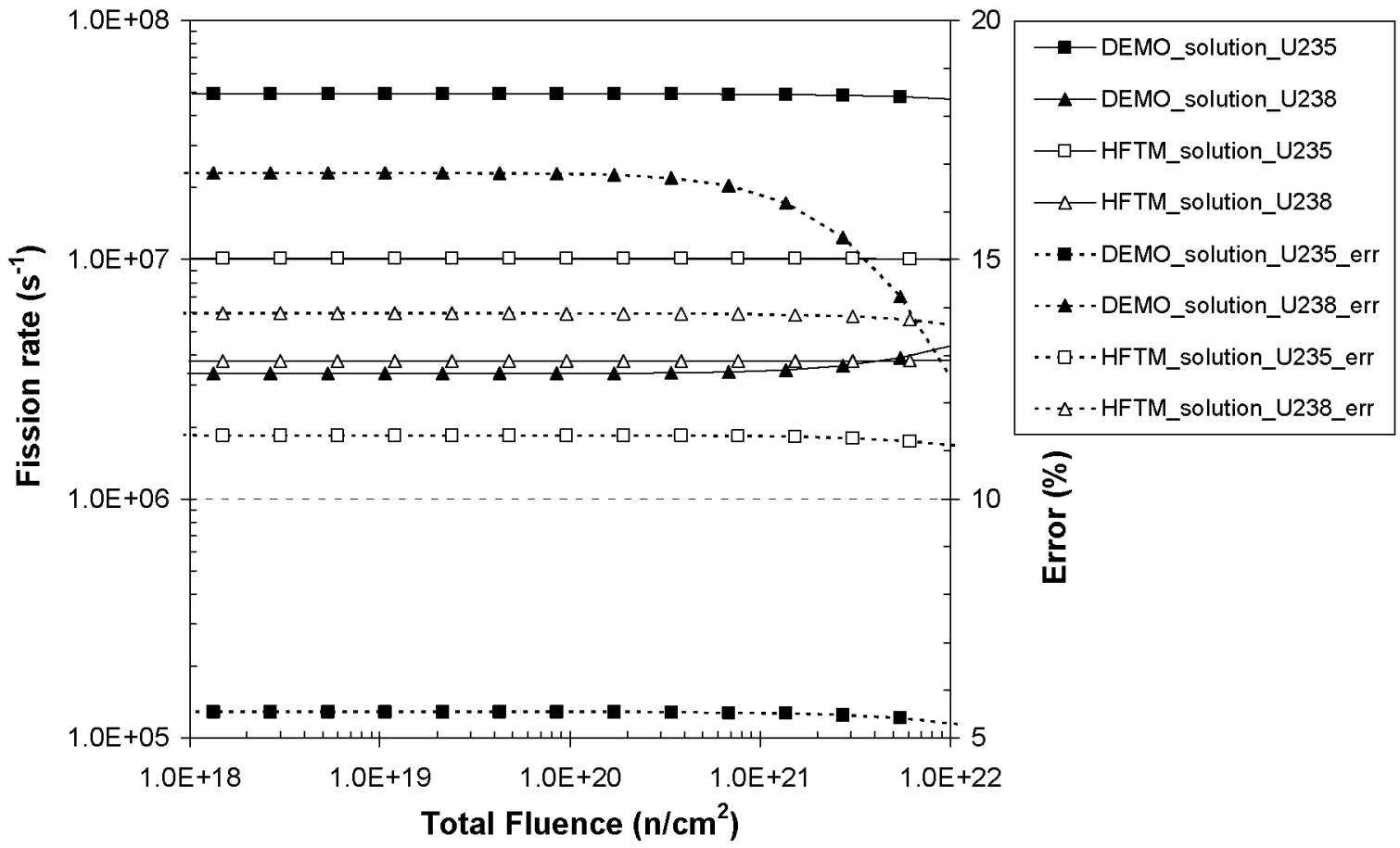

Fig. 11. Fission rates and relative error (in \%) for different U235 and U238 deposits in DEMO and HFTM/IFMIF neutron environments. 
that when evaluating cross-sections, the quantity $\log \left(\sigma_{\text {expt }} / \sigma_{\text {calc }}\right)$ was approximately normally distributed. Consequently, it can be said that for any given cross-section $\sigma$ we can define the random variable $\log (\sigma / \bar{\sigma})$ that follows a normal distribution with mean 0 and variance $\Delta_{\mathrm{EXP}}^{2}$. This well known distribution is equivalent to a normal distribution when $\Delta$ is small. [18]
Using this uncertainty information, and sampling with the adequate probability distribution, we obtain the transition matrix, A. With this A matrix, ACAB computes the vector of nuclides $(N)$ and response quantities $\left(R_{\text {tot }}, S_{\text {fast }}, \ldots\right)$ per nuclide. Repeating this sequence, it is possible to get a sample of vectors and, from this sample; we can estimate the mean, variance, etc. of the nuclide

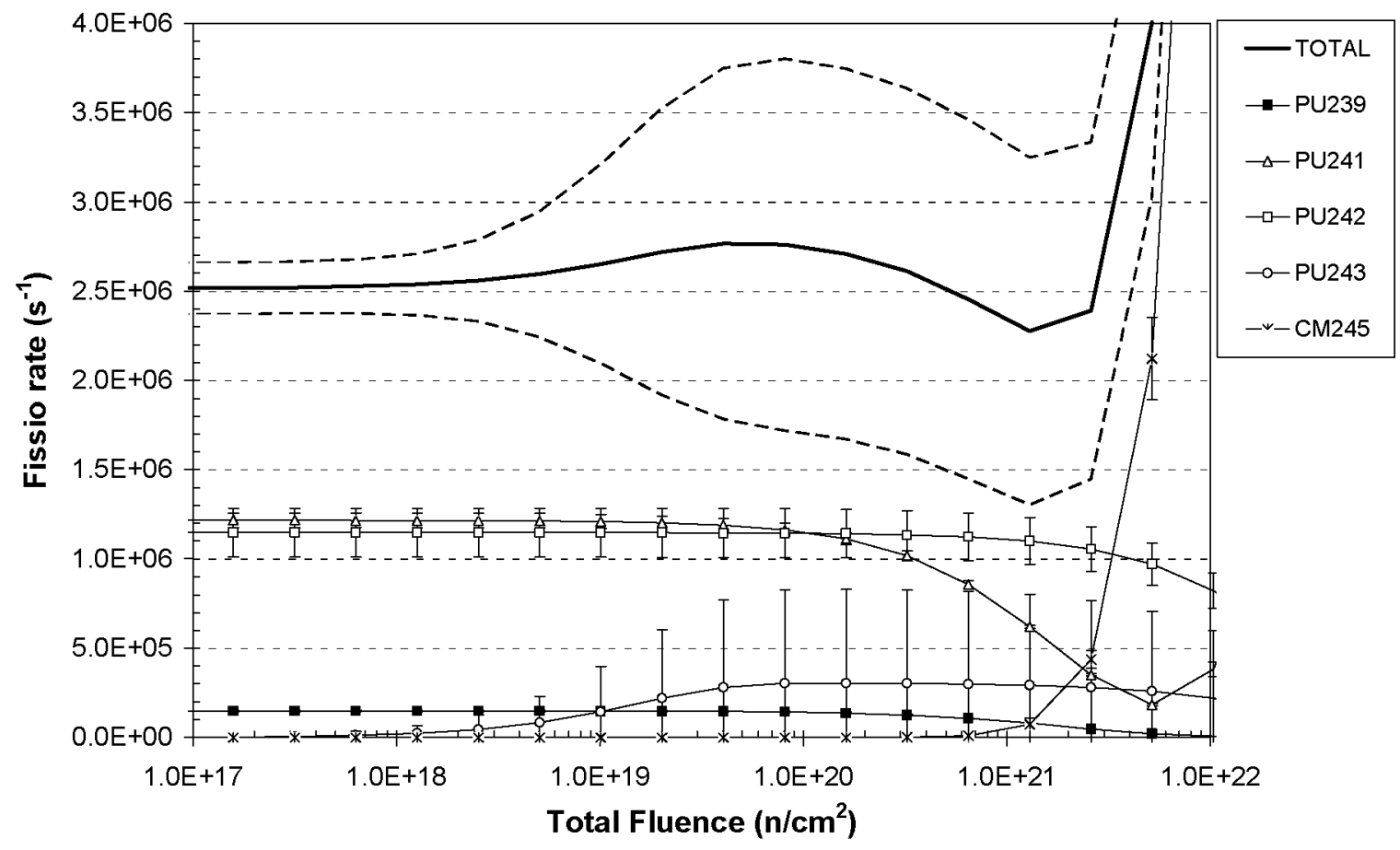

Fig. 12. Contribution and error bars (one standard deviation) of each isotope in the total fission rate for a deposit of Pu242\#2 (see Table 1 for initial composition) in a typical high flux thermal neutron environment (BR2).

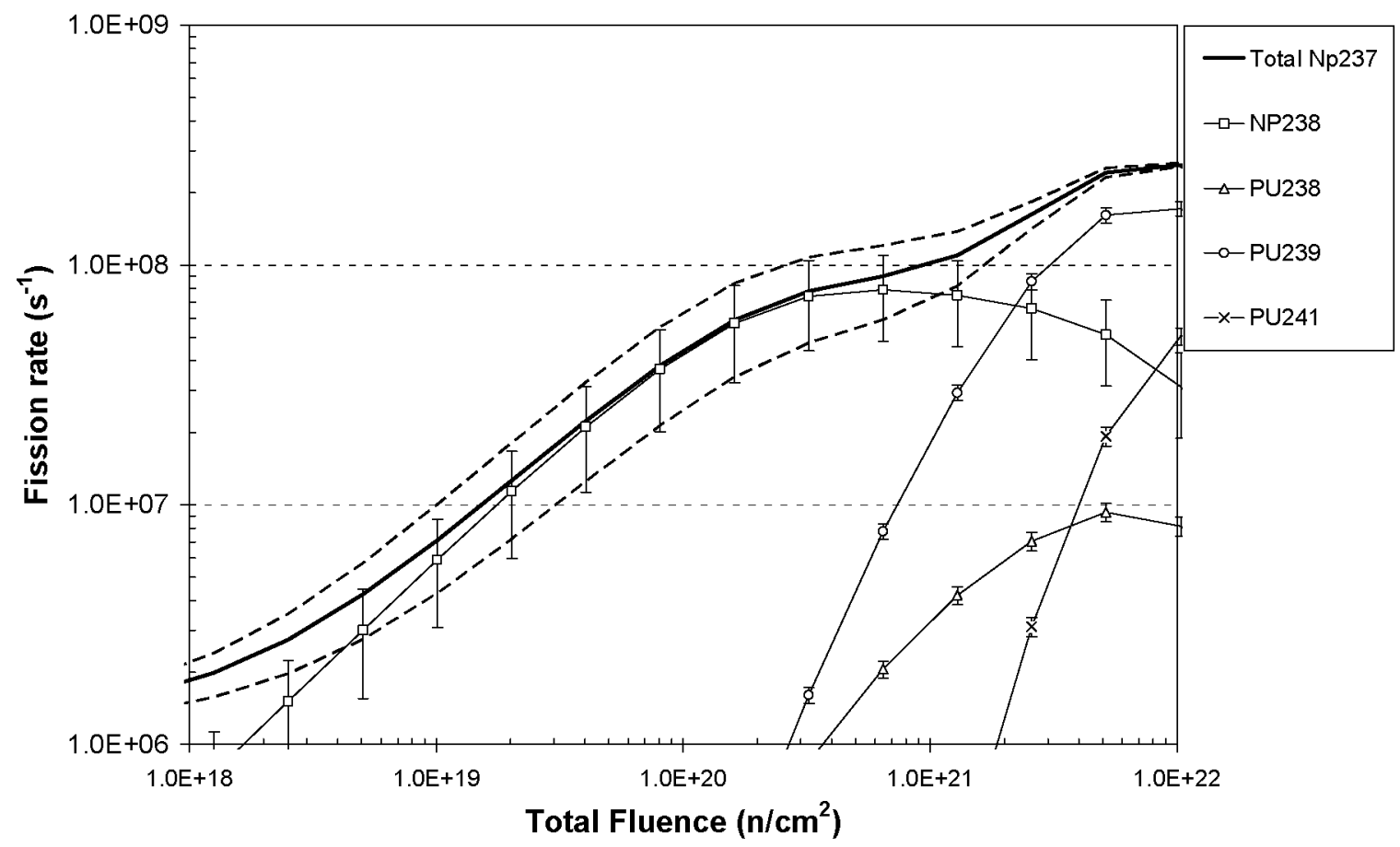

Fig. 13. Total fission rate and error bars (one standard deviation) for initially pure Np237 deposit irradiated in a high flux thermal environment (e.g. BR2). Contributions of each isotope to the total fission rate and errors are shown. 
and response quantities distribution. The uncertainty assessment has been computed by $A C A B$ code as a function of the neutron fluence. A 1000 histories sample size is found appropriate for this application.

Figs. 9 and 10 show the relative error in fission rates (in \%) and sensitivity to fast neutrons (in \%) for different pure and solution deposits in BR2. For deposits of Pu242 and Np237 large uncertainty values were found. These uncertainties are mainly due to the uncertainty in fission cross-section of ${ }^{243} \mathrm{Pu}(\sim 118 \%)$ and ${ }^{238} \mathrm{~Np}(\sim 47 \%)$ poorly known. For other deposits, uncertainties remain below $5 \%$ for fission rate and $18 \%$ for $S_{\text {fast }}$ up to fluences of $10^{22} \mathrm{n} / \mathrm{cm}^{2}$.

In Fig. 11, we have calculated the fission rate and relative error (in \%) for the deposits of U235 and U238 in the HFTM/IFMIF and DEMO neutron environments. Those values are nearly constant up to a neutron fluence of $10^{22} \mathrm{n} / \mathrm{cm}^{2}$. Relative errors are between $14 \%$ and $17 \%$ for U238 and between $5 \%$ and $15 \%$ for U235.

Figs. 12 and 13 show the contribution of each isotope and error bars for one standard deviation for deposit \#2 of Pu242 and deposit of Np237 in BR2, respectively.

In Fig. 12, the total fission rate with its error (one standard deviation) is illustrated, as well as the contribution of each isotope to the total fission rate with its error. The main contributors to the total fission rates below $10^{21} \mathrm{n} / \mathrm{cm}^{2}$ are ${ }^{241} \mathrm{Pu}$ and ${ }^{242} \mathrm{Pu}$. Contribution of ${ }^{245} \mathrm{Cm}$ is not significant below $10^{21} \mathrm{n} / \mathrm{cm}^{2}$, but it reaches $\sim 80 \%$ at $10^{22} \mathrm{n} / \mathrm{cm}^{2}$. Regarding errors in fission rates, below $10^{21} \mathrm{n} / \mathrm{cm}^{2}$, the main contributor to the total error is ${ }^{243} \mathrm{Pu}$. However, at higher fluence, the total uncertainty in fission rate $(\sim 15 \%)$ is mainly due to ${ }^{245} \mathrm{Cm}$. This is due to an isotopic relative error of ${ }^{245} \mathrm{Cm}$ of about $11 \%$ even if a fission cross-section uncertainty of ${ }^{245} \mathrm{Cm}$ as low as $4.5 \%$ can be found.

In Fig. 13, the total fission rate and error for initially pure Np237 irradiated in BR2 are presented. Contributions and errors for each isotope are also illustrated. Below $10^{21} \mathrm{n} / \mathrm{cm}^{2}$, it is shown the importance of ${ }^{238} \mathrm{~Np}$ uncertainty, with a relative error in fission cross-section of $\sim 47 \%$, and only an error of $\sim 5 \%$ in its concentration at $10^{20} \mathrm{n} / \mathrm{cm}^{2}$.

\section{Conclusions}

In this paper, we present an assessment of fissionable material behaviour in three neutron scenarios with different degrees of hardness (BR2, DEMO, and IFMIF). The evolution of fission rates as a function of the fluence for some potential/realistic deposits or solutions are predicted as well as other parameters having influence on the FC behaviour for a long-term performance (sensitivities to fast neutrons, total radioactivity, xenon prediction, and spectral history effect in BR2 due to changes in temperature). We conclude that in BR2, the fission rates are stable with deposits of Pu242 up to fluences as high as $10^{22} \mathrm{n} / \mathrm{cm}^{2}$ as well as satisfying high values of $S^{\text {fast }}$. For DEMO and IFMIF, fission rates remain stable for the complete set of deposits.

Concerning uncertainty calculation due to uncertainties in activation cross-sections we have analyzed for different neutron spectra the global uncertainty in the detector response. A Monte Carlo technique implemented in $A C A B$ code has been used to propagate those uncertainties during the irradiation time. In BR2, large uncertainties were found in deposits of Np237 and Pu242 due to uncertainties in fission cross-sections of ${ }^{238} \mathrm{~Np}$ and ${ }^{243} \mathrm{Pu}$, respectively. In addition, we have found that the uncertainty in the contribution of ${ }^{245} \mathrm{Cm}$ at high fluences in the deposits of Pu242 is mainly due to the uncertainty in its inventory. For other deposits, uncertainties remain below $5 \%$ for fission rates up to fluences of $10^{22} \mathrm{n} / \mathrm{cm}^{2}$. In the HFTM/IFMIF and DEMO, we found relative errors in fission rates between $5 \%$ and $17 \%$.

In conclusion, the knowledge of the evolution of these uncertainties can help to better understand the expected responses of fission chambers.

\section{Acknowledgements}

This work has been performed under Plan Nacional $I+D+I$ 2008-2011, ENE2008-06403-C06-06/FTN, Ministerio de Ciencia e Innovación, Spain and EU keep-in touch Program on IFE.

\section{References}

1] D. Rapisarda, A. García, O. Cabellos, et al., Fusion Eng. Design 84 (2009) 1570

[2] A. Letourneau, S. Breaud, O. Bringer, et al., Recent developments on micrometric fission chambers for high neutron fluxes, in: Proceedings of Advancements in Nuclear Instrumentation, Measurement Methods and their Applications, 2009, Marseille, France.

[3] P. Filliatre, L. Oriol, C. Jammes, L. Vermeeren, Nucl. Instr. and Meth. A 593 (2008) 510 .

[4] U. Fischer, S. Simakov, U.v. Möllendorff, P. Pereslavtsev, P. Wilson, Fusion Eng. Design 69 (2003) 485

[5] J. Sanz, O. Cabellos, N. García-Herranz, ACAB-2008, ACtivation ABacus Code V2008, NEA Data Bank (NEA-1839), 2009.

[6] R.A. Forrest, The European Activation System: EASY-2007 overview. UKAEA FUS 533, 2007

[7] R.A. Forrest, FISPACT-2007: User manual, Culham Report UKAEA FUS 534, 2005.

[8] M. Fadil, Ch. Blandin, S. Christophe, et al., Nucl. Instr. and Meth. Phys. Res. A 476 (2002) 313.

[9] S.P. Chabod, G. Fioni, A. Letourneau, F. Marie, Nucl. Instr. and Meth. Phys. Res. A 566 (2006) 633

[10] L. Vermeeren, On-line fast flux measurements in the BR2 reactor, SCK CEN, Scientific Report 2007. 〈www.sckcen.be

[11] S.P. Chabod, Nucl. Instr. and Meth. A 598 (2009) 578.

[12] O. Pujade, A. Lebrun, Nucl. Instr. and Meth. A 433 (1999) 673.

[13] L. Oriol, C.h. Blandin, S. Breaud, L. Vermeeren, M. Wéber, In-pile CFUZ53 Subminiature fission chambers qualification in BR2 under PWR condition, in: Proceedings of Joint TRTR-IGORR Meeting, Gaithersburg, MD, USA, September $12-16,2005$

[14] V.E. Viola, K. Kwiatkowski, M. Walker, Phys. Rev. C 31 (1985) 1550.

[15] SRIM 2008, available at 〈http://www.srim.org〉

[16] A. Bail, O. Serot, H. Faust, et al., Mass yield, isotopic yield and kinetic energy distributions for heavy masses in thermal neutron induced fission, in: Proceedings of International Conference on Nuclear Data for Science and Technology 2007, DOI:10.1051/ndata:07348.

[17] H. Hiruta, G. Palmiotti, M. Salvatores, R. Arcilla Jr., P. Obložinský, R.D. McKnight, Nucl. Data Sheets 109 (2008) 2801

[18] N. García-Herranz, O. Cabellos, J. Sanz, J. Juan, J.C. Kuijper, Ann. Nucl. Energy 35 (2008) 714 